\title{
The effects of plasma treatment on bacterial biofilm formation on vertically-aligned carbon nanotube arrays
}

Article in RSC Advances · January 2015

DOI: 10.1039/c4ra08187k

CITATIONS

8

8 authors, including:
READS

104

\section{Samuel Yick}

The Commonwealth Scientific and Industrial .. 27 PUBLICATIONS 206 CITATIONS SEE PROFILE

\section{Anthony Bruce Murphy}

The Commonwealth Scientific and Industrial ..

247 PUBLICATIONS 5,516 CITATIONS

SEE PROFILE

\section{Anne Mai-Prochnow}

The Commonwealth Scientific and Industrial ... 18 PUBLICATIONS 444 CITATIONS

SEE PROFILE

\section{Kostya Ostrikov}

Queensland University of Technology 630 PUBLICATIONS 11,445 CITATIONS

SEE PROFILE

Some of the authors of this publication are also working on these related projects: 


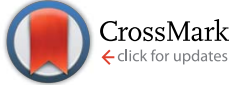

Cite this: RSC Adv., 2015, 5, 5142

Received 6th August 2014

Accepted 27th November 2014

DOI: $10.1039 / \mathrm{c} 4 \mathrm{ra0} 0187 \mathrm{k}$

www.rsc.org/advances

\section{The effects of plasma treatment on bacterial biofilm formation on vertically-aligned carbon nanotube arrays $\uparrow$}

\author{
Samuel Yick, $\dot{t}^{\mathrm{ab}}$ Anne Mai-Prochnow, $\dot{t}^{\mathrm{a}}$ Igor Levchenko, $\dot{t}^{\mathrm{ab}}$ Jinghua Fang, ${ }^{\text {ac }}$ \\ Michelle K. Bull, ${ }^{d}$ Mark Bradbury, ${ }^{d}$ Anthony B. Murphy ${ }^{\mathrm{a}}$ and Kostya (Ken) Ostrikov*abe \\ Carbon nanotubes (CNTs) can be fabricated with an ordered microstructure by controlling their growth \\ process. Unlike dispersed carbon nanotubes, these vertically-aligned arrays have the ability to support or \\ inhibit bacteria biofilms. Here, we show that by treating the carbon nanotube arrays with plasma, \\ different effects on biofilms of Gram-positive (Bacillus subtilis, Staphylococcus epidermidis) and Gram- \\ negative bacteria (Escherichia coli, Pseudomonas aeruginosa) can be observed.
}

\section{Introduction}

Bacteria are known to enable many chemical processes within the biosphere. The majority of bacteria form aggregates imbedded in an extracellular matrix, commonly referred to as a biofilm. ${ }^{1}$ These structures enable the cell to exhibit enhanced tolerance towards adverse environmental stresses; therefore there has been growing interest in utilizing biofilms for biotechnological applications. ${ }^{2}$ Recently, novel applications such as waste water treatment, ${ }^{3-5}$ starch hydrolysis, ${ }^{6}$ catalytic conversion, ${ }^{7,8}$ microbial fuel cell, ${ }^{9}$ and antifouling coatings have been realised by using cultivated biofilms. ${ }^{\mathbf{1 0 , 1 1}}$ However, in order for bacteria to be effectively exploited, platforms which can harness their output while maintaining the viability and activity within the biofilms need to be developed.

Of the many different platforms, contemporary interest in materials with nanostructures have grown considerably. Nanostructures possess surfaces at length scales which are relevant to biological systems. Therefore, controlling the interactions between the surface of nanostructures with cellular organisms can have vital implications for a host of fields such as in

${ }^{a}$ Plasma Nanoscience Laboratories, Manufacturing Flagship, Commonwealth Scientific and Industrial Research Organisation (CSIRO), P.O. Box 218, Lindfield, NSW 2070, Australia. E-mail: kostya.ostrikov@csiro.au

${ }^{b}$ Complex Systems, School of Physics, The University of Sydney, Sydney, NSW 2006, Australia

${ }^{c}$ School of Physics, University of Melbourne, Parkville, VIC 3010, Australia

${ }^{d}$ Food and Nutrition Flagship, Commonwealth Scientific and Industrial Research Organisation (CSIRO), P.O. Box 52, North Ryde, NSW 1670, Australia

${ }^{e}$ School of Chemistry, Physics, and Mechanical Engineering, Queensland University of Technology, Brisbane, QLD 4000, Australia

$\dagger$ Electronic supplementary information (ESI) available. See DOI: 10.1039/c4ra08187k

\$ Samuel Yick, Anne Mai-Prochnow and Igor Levchenko made equal contributions to the research reported in this article. medicine, ${ }^{\mathbf{1 2}, \mathbf{1 3}}$ agrochemical industries, pharmaceutics, ${ }^{\mathbf{1 4}}$ and energy management. ${ }^{15}$ By exploiting these nanomaterials, antimicrobial surfaces and sophisticated drug delivery systems have been developed. ${ }^{16,17}$ These examples show the usefulness of nanostructures in bio-related applications. In particular, highly-organized vertically-aligned carbon nanotubes (CNTs) are among the most attractive nanomaterials for biologically-active surfaces. ${ }^{18-20}$ CNTs are particularly attractive as they demonstrate outstanding physical properties and tunable morphologies. Furthermore, their surface characteristics can be altered through various post-treatment techniques, such as chemical modification and plasma processing. ${ }^{21,22}$ By controlling the process conditions, CNTs can be present as individual tubes, two-dimensional percolated networks or as three-dimensional vertically-aligned arrays. Compared to non-aligned structures, CNT arrays are of specific interest due to their larger available surface area, higher packing density and controllable microstructure. However, while the physical attributes of these arrays can be tuned with relative ease, an understanding of how their materials properties can affect the bacteria viability and biofilm formation remains elusive.

In this paper, we studied the relationship between the surface chemistry of vertically-aligned CNTs and its ability to support biofilm formation of various microorganisms. In particular, we show that vertically-aligned CNT arrays treated with inductively-coupled plasma (ICP) can demonstrate several very important properties, namely, (i) the selective support of biofilm towards Escherichia coli, Pseudomonas aeruginosa, Bacillus subtilis and Staphylococcus epidermidis; (ii) the ability to retain morphological integrity of the nanostructure in the presence of microbes and liquid medium; (iii) and the ability to exhibit biocompatibility. 


\section{Materials and methods}

\subsection{Growth of CNT arrays}

Vertically-aligned CNT arrays were synthesized via a thermal chemical vapour deposition (TCVD) process. A $10 \mathrm{~nm}$ layer of $\mathrm{Al}_{2} \mathrm{O}_{3}$ was reactively sputtered on the thermally-oxidized $\mathrm{Si}$ wafer. After that, $1.8 \mathrm{~nm}$ of Fe was deposited onto the substrate by magnetron sputtering. The prepared substrates were loaded into a quartz tube of a thermal furnace (MTI, OTF-1200). Then, the temperature in the furnace was ramped up to $700{ }^{\circ} \mathrm{C}$ under a constant flow of $\mathrm{Ar}(800 \mathrm{sccm})$ at atmospheric pressure. Once the working temperature was reached, a mixture $\mathrm{H}_{2}+\mathrm{C}_{2} \mathrm{H}_{2}$ was also introduced at 240 and $56 \mathrm{sccm}$, respectively. After a certain growth time (typically $10 \mathrm{~min}$ ), the supply of $\mathrm{H}_{2}$ and $\mathrm{C}_{2} \mathrm{H}_{2}$ was terminated, and the samples were cooled down to room temperature under a continuous Ar flow. This process results in the growth of the CNT arrays. A schematic of the furnace and further details of the process can be found in the ESI. $\dagger$

\subsection{Plasma modification of CNT arrays}

The as-grown CNT arrays were treated with Ar plasmas generated via an Inductively-Coupled Plasma (ICP) system (Fig. 1d). Briefly, the sample was first inserted into a vacuum chamber. 20 sccm of Ar was flowed into the chamber and the pressure was set to $5 \mathrm{~Pa}$. The sample was treated by argon plasmas ignited at power ranging from 200 to $1000 \mathrm{~W}$ for 2 minutes.

\subsection{Bacteria experiment}

Bacillus subtilis, Escherichia coli, Staphylococcus epidermidis and Pseudomonas aeruginosa were grown overnight in nutrient broth (NB) broth at $37^{\circ} \mathrm{C}$ to an optical density of 1 . A $5 \mu \mathrm{L}$ aliquot of overnight culture was inoculated into $1 \mathrm{~mL}$ of fresh $\mathrm{NB}$ broth in a well of a 24-well plate; each well also contained a $1 \mathrm{~cm}^{2}$ piece of CNT array sample. The 24-well plate was incubated for 16 hours at $37^{\circ} \mathrm{C}$ with slight shaking ( $\left.80 \mathrm{rpm}\right)$. The areal coverage of the colonies was calculated using ImageJ. To determine the viability of the bacteria, aliquots from the incubated wells were taken for live cell counts to determine the number of colony forming units (CFU).

\subsection{Flow cytometry}

Bacteria from E. coli and B. subtilis biofilms formed on the nanostructures were used in the flow cytometry measurements. Bacterial biofilms were scraped off the CNT arrays using a sterile metal spatula and dispersed into $2 \mathrm{~mL}$ of phosphatebuffered saline (PBS). Samples were sonicated for $1 \mathrm{~min}$ to break up cell clumps before staining with LIVE/DEAD ${ }^{\circledR}$ BacLight $^{\mathrm{TM}}$ (Invitrogen) according to the manufacturer's instructions. A 6-hour fresh culture of each strain was diluted into the PBS. One half of this culture was used as a live control and stained with each of the components (SYTO 9 and PI) separately. The other half was heat killed by exposing the culture to $90{ }^{\circ} \mathrm{C}$ for $10 \mathrm{~min}$ in a water bath before staining.

\subsection{Preparing biological samples for SEM}

In order to enable the utilisation of electron microscopic analysis, the bacteria laden samples were prepared with critical point drying (CPD). The samples were first immersed in 100\% ethanol, then transferred to the CPD chamber (BAL-TEC CPD030 Critical Point Dryer) and dried using liquid $\mathrm{CO}_{2}$ for 3 hours at the critical point $\left(+31.1^{\circ} \mathrm{C}, 1000 \mathrm{psi}\right)$. This process allows the structural integrity of both the bacteria and CNT arrays to be preserved in a high vacuum environment.

\subsection{Material characterisation}

Field-emission scanning electron microscopy (FE-SEM; Zeiss Auriga) operated at an electron acceleration voltage of $5 \mathrm{kV}$ and a working distance of $2.6 \mathrm{~mm}$. Transmission electron microscopy (TEM; JOEL 2200) was operated at electron beam energy of $200 \mathrm{keV}$. X-ray photoelectron spectroscopy (XPS) measurements were taken using the PHI Specs Sage 150 system, where the Mg
A

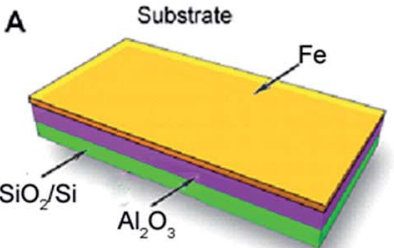

B Metal fragmentation

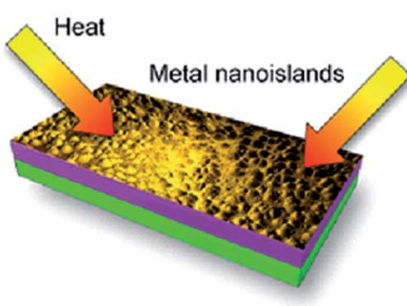

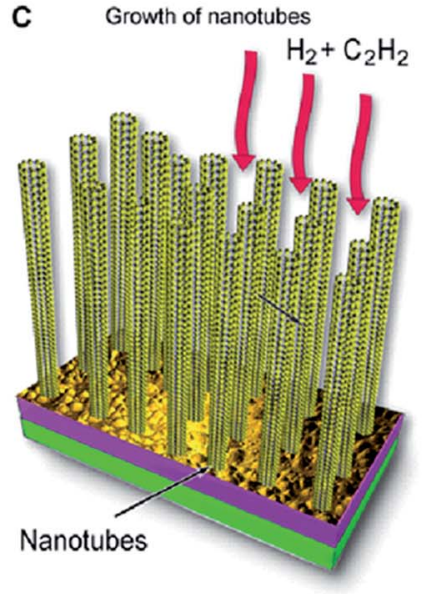
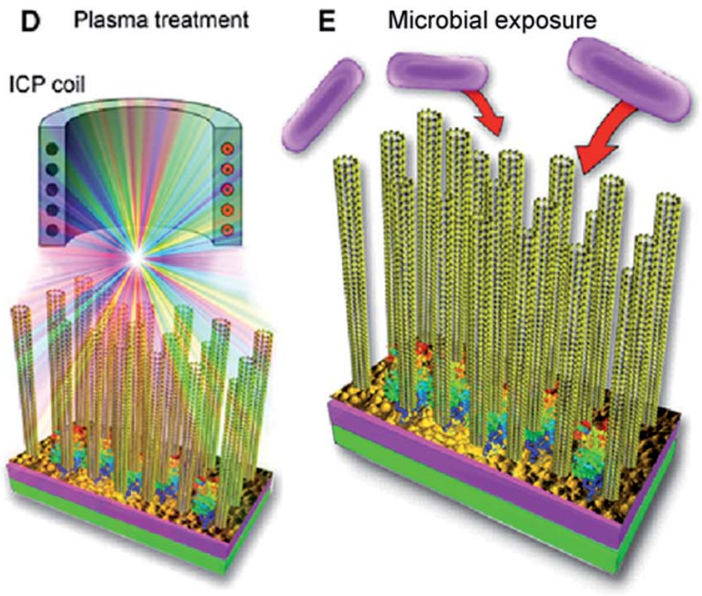

Fig. 1 Experiment overview: (a) structure of the substrate prepared for the formation of carbon nanotube arrays. (b) Fragmentation of metal catalyst to form catalytic-active island structures. (c) Growth of CNTs on the fragmented metal catalyst. (d) Plasma treatment. (e) Schematic of microbial incubation with the prepared structure. 
$\mathrm{K} \alpha$ (at $1253.6 \mathrm{eV}$ ) line was used as the excitation source. The Raman spectra were collected by Renishaw InVia confocal Raman microscope system with a $50 \times$ objective lens. Samples were excited with a $514 \mathrm{~nm}$ laser at power of $\sim 1.5 \mathrm{~mW}$ and a spot diameter of $\sim 1 \mu \mathrm{m}$.

\section{Results and discussion}

\subsection{CNT array structures}

The scanning electron microscopy (SEM) images of the structures used in our studies are shown in Fig. 2. The topography of the CNT arrays is comprised of slightly entangled nanotubes with submicron voids present. These voids are impermeable to the bacteria due to their physical dimensions. From examining the cross-sectional SEM micrographs depicted in Fig. 2a and b one can conclude that the CNT arrays possessed an average height of $20 \mu \mathrm{m}$ and an estimated density of $3-5 \times 10^{10}$ tubes per $\mathrm{cm}^{2}$. Note that the nanotubes are not straight but slightly bent and clogged. Transmission electron microscopy (TEM) images showed that the pristine CNTs had a typical diameter of $10 \mathrm{~nm}$ with 5-10 walls visible. The clearly distinguishable lattice fringes observed in the pristine sample indicate their high graphitic ordering (Fig. 2e and f). After the plasma treatment at $1000 \mathrm{~W}$, though the diameter of the tubes remained the same, the lattice fringes and the internal void were no longer observable (Fig. $2 \mathrm{~g}$ and $\mathrm{h}$ ). Furthermore, the tip of the nanotube appears to be partially opened by the treatment, affecting surface roughness.

The changes induced to the graphitic structure by the plasma treatment were further examined using Raman spectroscopy. The spectra (see Fig. S3, ESI $\dagger$ for the spectra and detailed description of the Raman results) displayed many features commonly associated with multi-walled CNTs, which include the D-peak at $1340 \mathrm{~cm}^{-1}$, the G-peak at $1570 \mathrm{~cm}^{-1}$ and the second-order Raman resonant feature (2D-peak) at $2680 \mathrm{~cm}^{-1}$. Even at a glance, the differences between the two spectra can be clearly observed. Most notably, the plasma treatment generated a shoulder featured at $1604 \mathrm{~cm}^{-1}$. This feature, commonly referred to as the $\mathrm{D}^{\prime}$ peak, is a defectinduced single-phonon intra-valley scattering process. This feature arises from perturbations within the $\mathrm{sp}^{2}$ lattice induced by voids and edge defects. Therefore, the emergence of the $\mathrm{D}^{\prime}$ feature indicates that the Ar plasma treatment created voids in the graphitic networks and edge defects through opening the tubes. These results from Raman and TEM analysis confirm that the plasma treatment led to the creation of voids and edge defects on the CNTs. These features allows the generation of chemically reactive sites without changing the morphology of the CNT arrays.

The chemical composition of the CNT array was quantified by X-ray photoelectron spectroscopy (XPS). Only carbon atoms were detected in the pristine sample, whereas oxygen atoms were also present in the $1000 \mathrm{~W}$ plasma-treated sample. From the detailed analysis of the XPS results (see Fig. S4, ESI $\dagger$ for further details) it was concluded that oxygen functionalization occurred once the plasma-treated CNT arrays had been exposed to air. As a result of the plasma functionalization, the atomic percentage of oxygen increased from $1.2 \%$ to $7.0 \%$. From this, it can be seen that the presence of defect sites allow the CNT arrays to be functionalized with oxygen-containing moieties once exposed to the atmosphere. This demonstrates that the plasma treatment was indeed able to modify the surface reactivity, while preserving the morphology of the CNT arrays.

\subsection{Bacteria experiment I: effects of bacteria type and plasma treatment on the biofilm formed on the CNT arrays}

To investigate biofilm formation on the CNT arrays, B. subtilis and $E$. coli were incubated with the pristine and plasma-treated
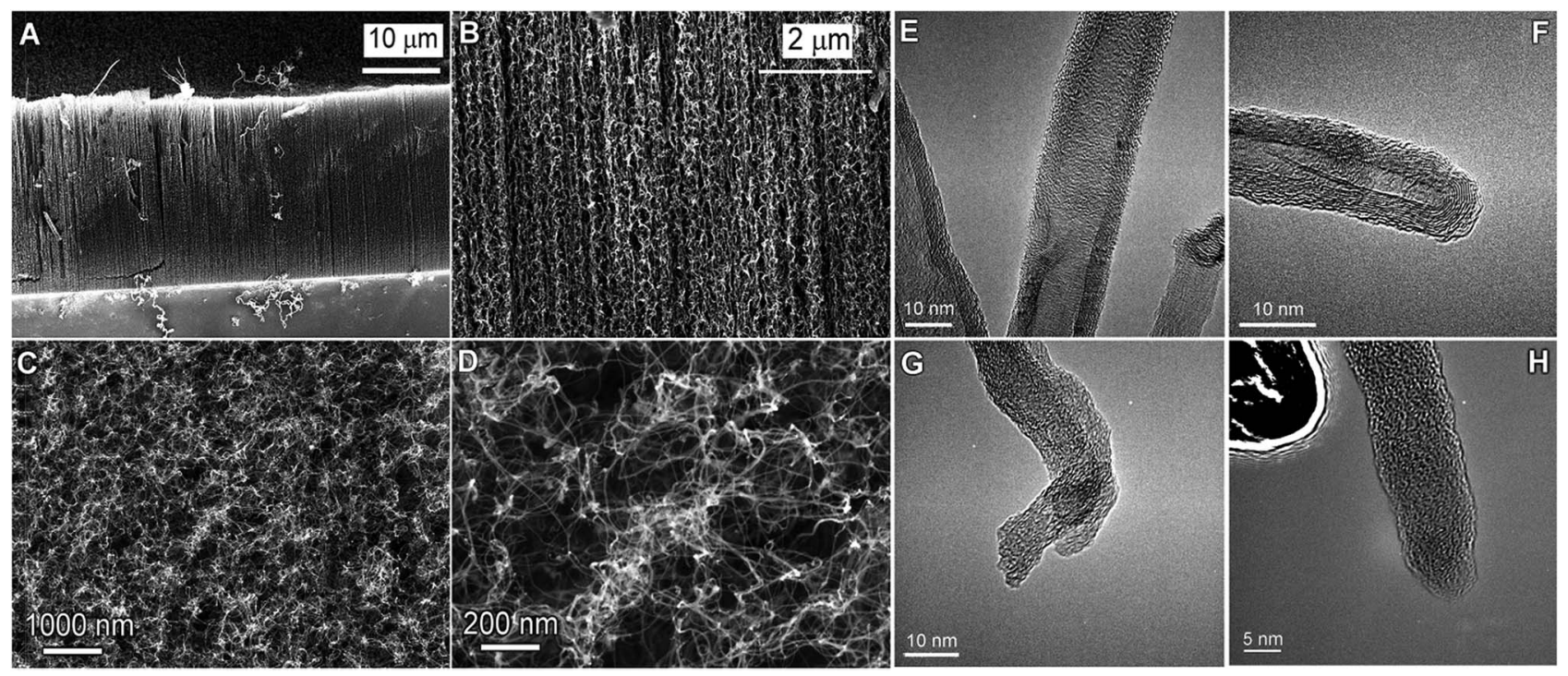

Fig. 2 SEM and TEM characterization of the carbon nanotube array: (a and b) low- and high-resolution SEM images of as-prepared nanotube array (side views). (c and d) Top views of the as-prepared nanotube array. (e and f) High-resolution TEM images depicting pristine nanotubes, and ( $\mathrm{g}$ and $\mathrm{h}$ ) nanotubes after a $1000 \mathrm{~W}$ plasma treatment. 

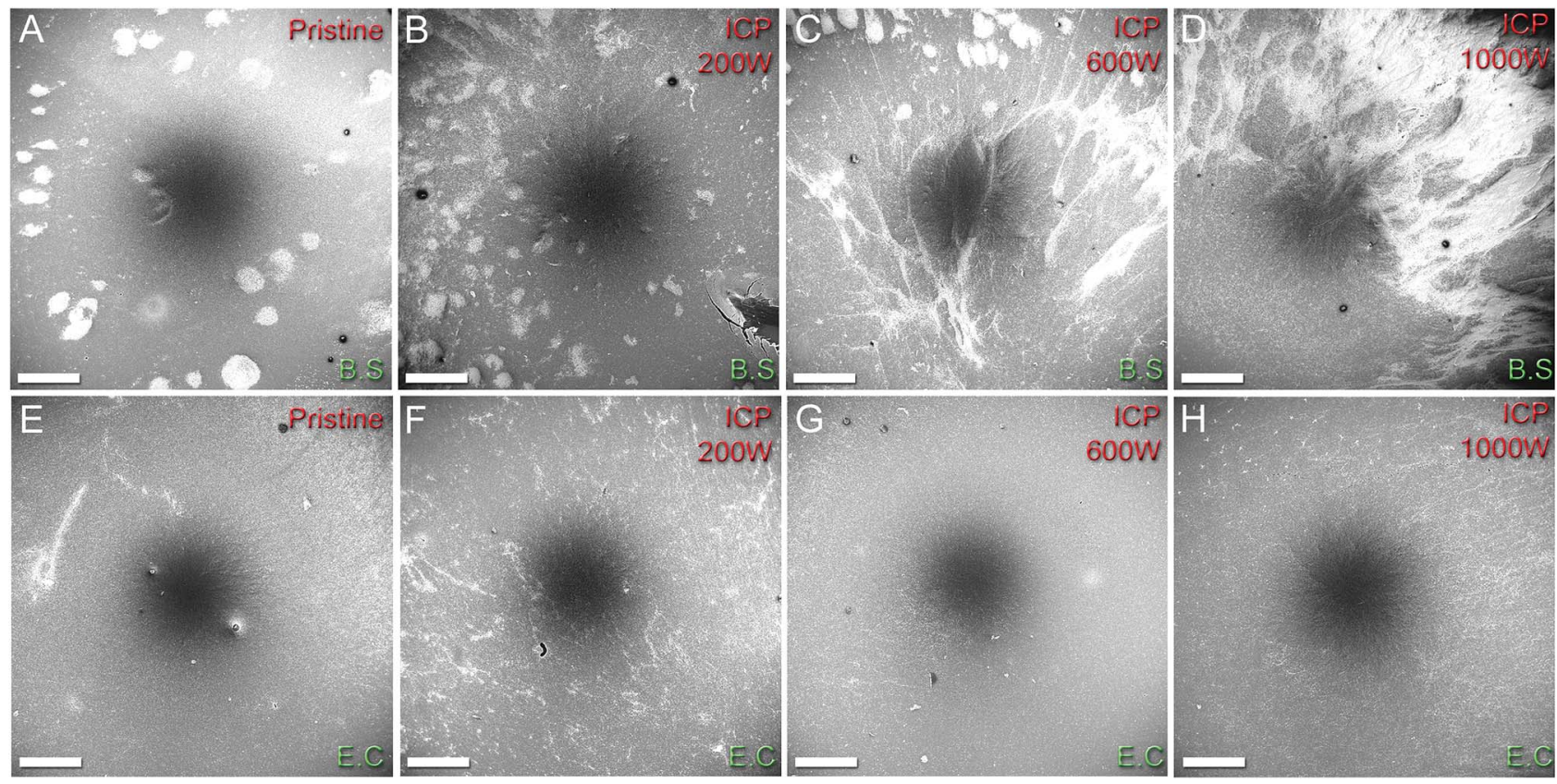

Fig. 3 SEM images of the bacterial colonies formed on the CNT arrays treated by plasma of different power. The top row depicts the colonies formed by $B$. subtilis whereas the bottom row represents the colonies formed by $E$. coli. All scale bars represent $400 \mu \mathrm{m}$.

CNT arrays. To observe bacterial attachment and biofilm formation, samples were visualized using SEM (Fig. 3). At a glance, the experiment demonstrated that the bacteria could not penetrate inside the nanotube array. Indeed, though voids are present, they were much smaller than the typical size of the bacteria $(\sim 2 \mu \mathrm{m})$, and thus the structure ensured sterile conditions within the interior of the array.

The ability of the bacteria to attach to the CNTs and to grow in the immediate surroundings of the CNTs was further examined. Though both bacteria were able to attach and form colonies on all CNT samples investigated, the size and density of these colonies differed by bacteria species and plasma treatment. For B. subtilis, only individual microcolonies and a few single cells were observed in the pristine sample. However, with increasing ICP power and thus increasing surface functionalization, the size and density of the microcolonies increased correspondingly. From these micrographs, B. subtilis biofilm achieved areal coverages of $6.18 \%, 11.6 \%, 17.5 \%$ and $37.0 \%$ for pristine, $200 \mathrm{~W}-, 600 \mathrm{~W}$ - and $1000 \mathrm{~W}$-treated CNT arrays, respectively. This result is in contrast with the often-observed phenomenon where a higher degree of functionalization leads to a higher degree of antibacterial activity. ${ }^{23} \mathrm{E}$. coli also attached onto the CNTs (Fig. 3e-h). However, unlike B. subtilis, colonies formed by $E$. coli on the pristine and plasma-treated samples looked similar in morphology. Areal coverages of the E. coli colonies on pristine, $200 \mathrm{~W}$-, $600 \mathrm{~W}$ - and $1000 \mathrm{~W}$-treated CNT arrays remained relatively consistent at $2.57 \%, 5.70 \%, 2.75 \%$ and $2.52 \%$, respectively. The variation in the biofilm-forming ability between $B$. subtilis and $E$. coli may be due to the differences in cell wall/membrane structure between the two bacteria, which resulted in varying response towards plasma-functionalized CNT arrays.
As shown in the XPS, the process of plasma treatment led to the attachment of oxygen containing moieties such as carboxyl, hydroxyl and carbonyl groups (Fig. S4 ESI $\dagger$ ). The presences of these functional groups can lead to a change to the surface potential of the CNTs. ${ }^{24}$ The presences of these changes can alter the process of biofilm formation through affecting the initial adherence of the cell onto the CNTs. ${ }^{25}$ From our experimental results, we observe differences in the biofilm formed by B. subtilis and E. coli on pristine and plasma-treated CNT arrays. It is possible that such variations were induced by the changes to the surface potential, as the physiological difference between B. subtilis and E. coli allows different modes of surface interaction. While it is entirely possible that the changed surface potential is sufficient to explain the differences observed between these bacteria, this may be an overly simplistic interpretation. The ability for bacteria to colonize a surface and form a biofilm depends on numerous aspects. ${ }^{26}$ Therefore, further studies will be needed to elucidate other factors which contribute to biofilm formation on carbon nanostructures.

\subsection{Bacteria experiment II: influence of plasma treatment on bacterial numbers}

The effects of the nanostructure and the plasma treatment on the growth of bacteria was investigated by quantifying the number of bacteria present in the supernatant surrounding the CNTs. Bacteria were incubated in a rich growth medium in the presence of CNTs for $24 \mathrm{~h}$ before being diluted and counted using colony forming units (CFU). In order to assess the samples, a control experiment where the bacteria were incubated in the absence of any CNT arrays was also conducted. For B. subtilis the control sample showed $2 \times 10^{8} \mathrm{CFU} \mathrm{mL}^{-1}$ 

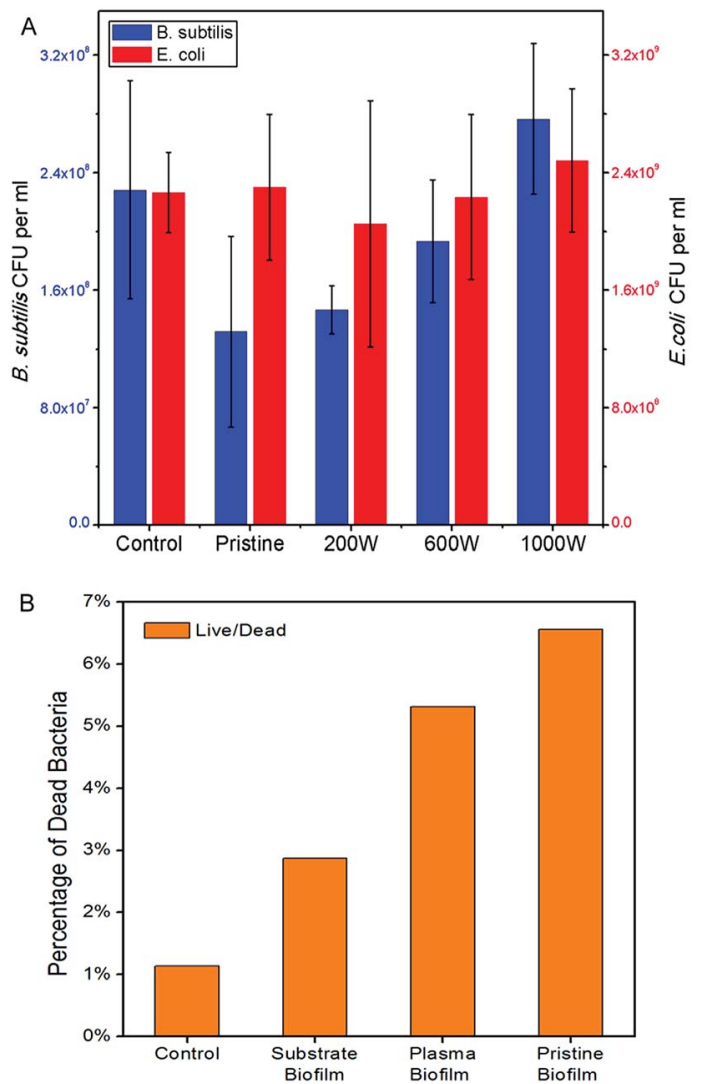

Fig. 4 Controlled growth of B. subtilis and E. coli on pristine and plasma-treated samples: (a) dependence of cell concentration on the discharge power. (b) The percentage of dead cells in the nanotubecontaining biofilm as established by flow cytometry. Bacteria samples were obtained from the biofilm formed on the control, catalyst-coated silicon wafer (substrate), and the CNT arrays.

(Fig. 4a). Bacteria grown in the presence of the pristine CNT

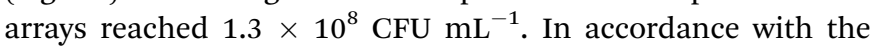
SEM pictures, where thicker biofilms were observed on the plasma-treated (functionalized) CNTs, the bacterial concentration was higher $\left(2.8 \times 10^{8} \mathrm{CFU} \mathrm{mL}^{-1}\right)$ for the cells grown in the presence of plasma-treated CNTs after $24 \mathrm{~h}$. Bacterial numbers reached levels similar to the control sample (Fig. 4a).

Interestingly, this trend was not observed when CNTs were incubated with Gram-negative $E$. coli. The control samples reached $2.5 \times 10^{8} \mathrm{CFU} \mathrm{mL}^{-1}$ after $24 \mathrm{~h}$ (Fig. $4 \mathrm{a}$ ). No significant decrease in cell numbers was observed when $E$. coli was incubated with pristine CNTs and only a small decrease was observed for the $200 \mathrm{~W}$ plasma-treated sample. A slight increase in cell numbers with increasing plasma treatment occurred; however, this was not as significant as for B. subtilis (Fig. 4a). This is consistent with our SEM results, where $E$. coli biofilms on pristine and functionalized samples appeared similar in morphology but an effect was observed for the B. subtilis samples on pristine CNTs. Gram-negative bacteria possess an outer membrane comprised of lipopolysaccharide, which makes them intrinsically more resistant to some antibiotics and physical forces. Similarly, the outer membrane of $E$. coli could be responsible for a higher resistance to CNT arrays as compared to $B$. subtilis. To confirm this observation of a selective interaction of CNTs depending on Gram type, another two organisms, $S$. epidermidis and $P$. aeruginosa, which are Gram-positive and Gram-negative respectively, were exposed to the plasma-treated CNT arrays (ESI Fig. S5 $\dagger$ ). Again, we observed the Gram-positive species exhibit a greater response to the plasma-treated substrate. While Gram-positive bacteria have a thicker cell wall, the outer membrane, which is only present in Gram-negative bacteria, was previously shown to be responsible for higher resistance to antibiotics and CNTs. ${ }^{27,28}$ Similarly, $E$. coli and $P$. aeruginosa were not influenced by the presence of the CNT arrays regardless of functionalization. In contrast, the growth of $B$. subtilis and S. epidermidis was inhibited by pristine CNT arrays. However, the array's impediment to bacterial growth subsided upon plasma treatment. Thus, we were able to show that bacterial inhibition of the CNT arrays could be finetuned by varying the plasma treatments.

\subsection{Bacteria experiment III: the biocompatibility of CNT arrays}

To understand the interaction between the CNT arrays and bacteria, a fluorescent staining technique was used in conjunction with flow cytometry. LIVE/DEAD® BacLight ${ }^{\mathrm{TM}}$ staining uses two intercalating dyes: the cell membrane permeant SYTO 9 to stain all bacterial cells green and the membrane-impermeant propidium iodide to counterstain only cells with a compromised cell membrane (i.e., dead cells). Following the excitation of the nucleic acid-bound dyes, flow cytometry was used to rapidly detect live (green) and dead (red) cells, and gave an indication of the viability state of bacteria grown in the presence of CNTs. More details on the flow cytometry can be found in ESI, section S6. $\uparrow$ Interestingly, the percentage of dead cells was about $6 \%$ in the pristine sample, while the control (no substrate) had 1\% dead cells (Fig. 4b). This was unexpected, as exposure to CNT often lead to a high percentage of dead bacterial cells. ${ }^{28,29}$

Our results indicated a selective interaction induced by the plasma process against different bacteria species. Three antibacterial mechanisms of CNTs have been proposed in the literature: oxidative stress (i), metal toxicity (ii), and physical piercing (iii). ${ }^{30}$ In our case, fatal oxidative stress was unlikely as the correlation between the extent of functionalization and the generation of reactive oxidative species (ROS) was weak. This could be seen in Fig. $4 \mathrm{a}$ as the plasma treatment became more extensive; the biocompatibility of the treated CNT arrays either increased or remained the same. This is a striking observation, as it is a common belief that functionalized CNTs are capable of higher ROS generation. ${ }^{31}$ However, prior studies have demonstrated that functionalized CNTs are able to quench and scavenge free radicals. ${ }^{32}$ This could explain our observation that enhanced plasma functionalization resulted in a higher biocompability, i.e. more bacterial biofilms.

Metal toxicity from catalyst residues is also unlikely to be a significant factor in the selective action of our CNT arrays, because $B$. subtilis incubated with the silicon wafer with catalyst had a much lower percentage of dead cells (Fig. 4b, substrate biofilm) when compared to both pristine and 
plasma-treated CNT arrays, which should have similar metal residues. Metal toxicity is often attributed to their generation of ROS, which cause fatal oxidative stress. In publications which spoke of the ROS-generating ability of metallic residues, they are often located in the intracellular space. ${ }^{33}$ It is highly likely that due to the surface bound nature of our catalyst, they are excluded from the intracellular space which leads to their passivity.

A direct physical piercing action is often the main cause for bacterial inhibition by CNTs and this could also be true for the CNT arrays in our study. From the flow cytometry analysis, the number of dead cells after contact with CNT arrays was higher as compared to the control sample. However, the total number of dead cells in the pristine CNT sample remained relatively small $(<7 \%)$ compared to the significant decrease in viable cells (CFU counts) observed. This relatively small number of dead cells in the pristine CNT array sample is in contrast to what is commonly observed when bacteria are exposed to dispersed CNTs. For example, Liu et al. reported E. coli and B. subtilis incubated with CNTs (dispersed by sodium cholate) resulted in a death rate of 80 and $90 \%$ respectively. ${ }^{29}$ The differences between the numbers of dead cells caused when bacteria were incubated in CNT suspensions versus CNT arrays could perhaps be understood by the mobility exhibited by dispersed CNTs. When CNTs are dispersed in a medium, they possess momentum and kinetic energy which allows them to pierce cells at a high rate. ${ }^{29}$ However, upon being fixed to a substrate such mobility is lost. This reduced their ability to pierce cellular membranes. Furthermore, it had been shown that graphitic surfaces are less capable of degrading the lipid membrane of bacteria if they are more hydrophilic. ${ }^{34}$ Thus, chemically functionalized (i.e. reduced hydrophobicity) CNT arrays affect cell viability less than the pristine arrays. Hence, the biocompatibility of CNT arrays can be enhanced by the plasma-assisted surface functionalization.

\section{Conclusions}

The work presented showed that CNT arrays can be considered as a viable biological platform. Unlike dispersed CNTs, they did not exhibit strong antibacterial activity and were selective towards the biofilm which they supported. We have further demonstrated that the process of plasma treatment causes vertically-aligned CNTs to exhibit dissimilar effects towards Gram-negative and Gram-positive bacteria. Gram-positive bacteria such as $B$. subtilis and $S$. epidermidis displayed enhanced biofilm formation and high CFU counts on the CNT arrays upon plasma treatment. However, E. coli and P. aeruginosa, which are Gram-negative bacteria, did not show much response to the presence of CNTs regardless of functionalization. We have attributed such differences to the physiological variation between the two bacteria types which responded differently to the presences of pristine and plasma-functionalized CNT arrays. This work demonstrates that CNT arrays can act as a viable platform for the control and cultivation of biofilms, which is necessary for novel applications.

\section{Acknowledgements}

This work was partially supported by CSIRO's OCE Science Leadership Research Program, CSIRO Sensors and Sensor Network TCP, the Australian Research Council and the University of Sydney.

\section{Notes and references}

1 T. Bjarnsholt, O. Ciofu, S. Molin, M. Givskov and N. Hoiby, Nat. Rev. Drug Discovery, 2013, 12, 791.

2 B. Halan, A. Schmid and K. Buehler, Appl. Environ. Microbiol., 2011, 77, 1563.

3 N. O. San, A. Celebioglu, Y. Tumtas, T. Uyar and T. Tekinay, RSC Adv., 2014, 4, 32249.

4 K.-C. Lee and B. E. Rittmann, Water Res., 2002, 36, 2040.

5 C. Accinelli, M. L. Saccà, M. Mencarelli and A. Vicari, Bioresour. Technol., 2012, 120, 180.

6 R. Gupta, P. Gigras, H. Mohapatra, V. K. Goswami and B. Chauhan, Process Biochem., 2003, 38, 1599.

7 K. J. Martin and R. Nerenberg, Bioresour. Technol., 2012, 122, 83.

8 C. Li and L.-K. Ju, RSC Adv., 2014, 4, 44026.

9 B. E. Logan, Nat. Rev. Microbiol., 2009, 7, 375.

10 R. de Nys and P. D. Steinberg, Curr. Opin. Biotechnol., 2002, 13, 244.

11 J. G. Burgess, K. G. Boyd, E. Armstrong, Z. Jiang, L. Yan, M. Berggren, U. May, T. Pisacane, A. Granmo and D. R. Adams, Biofouling, 2003, 19, 197.

12 X.-R. Li, B. Wang, J.-J. Xu and H.-Y. Chen, Nanoscale, 2011, 3, 5026.

13 H. Mao, R. Cai, N. Kawazoe and G. Chen, Nanoscale, 2014, 6, 1552.

14 V. D'Britto, H. Kapse, H. Babrekar, A. A. Prabhune, S. V. Bhoraskar, V. Premnath and B. L. V. Prasad, Nanoscale, 2011, 3, 2957.

15 M. I. Ruiz, C. I. Sanchez, R. G. Torrres and D. R. Molina, J. Braz. Chem. Soc., 2011, 22, 2337.

16 K. Vasilev, V. Sah, K. Anselme, C. Ndi, M. Mateescu, B. Dollmann, P. Martinek, H. Ys, L. Ploux and H. J. Griesser, Nano Lett., 2010, 10, 202.

17 H. Pandey, V. Parashar, R. Parashar, R. Prakash, P. W. Ramteke and A. C. Pandey, Nanoscale, 2011, 3, 4104.

18 A. Kondyurin, I. Levchenko, Z. J. Han, S. Yick, A. MaiProchnow, J. Fang, K. Ostrikov and M. M. M. Bilek, Carbon, 2013, 65, 287.

19 K. Ostrikov, I. Levchenko, U. Cvelbar, M. Sunkara and M. Mozetic, Nanoscale, 2010, 2, 2012.

20 O. Volotskova, I. Levchenko, A. Shashurin, Y. Raitses, K. Ostrikov and M. Keidar, Nanoscale, 2010, 2, 2281.

21 I. Levchenko, K. Ostrikov, D. Mariotti and V. Švrček, Carbon, 2009, 47, 2379.

22 S. Kumar, I. Levchenko, K. Ostrikov and J. A. McLaughlin, Carbon, 2012, 50, 325.

23 H. Chen, B. Wang, D. Gao, M. Guan, L. Zheng, H. Ouyang, Z. Chai, Y. Zhao and W. Feng, Small, 2013, 9, 2735. 
24 H. Hu, Y. Ni, V. Montana, R. C. Haddon and V. Parpura, Nano Lett., 2004, 4, 507.

25 L. Hall-Stoodley, J. W. Costerton and P. Stoodley, Nat. Rev. Microbiol., 2004, 2, 95.

26 G. O'Toole, H. B. Kaplan and R. Kolter, Annu. Rev. Microbiol., 2000, 54, 49.

27 H. Nikaido, Clin. Infect. Dis., 1998, 27, S32.

28 A. R. Deokar, L.-Y. Lin, C.-C. Chang and Y.-C. Ling, J. Mater. Chem. B, 2013, 1, 2639.

29 S. Liu, L. Wei, L. Hao, N. Fang, M. W. Chang, R. Xu, Y. Yang and Y. Chen, ACS Nano, 2009, 3, 3891.
30 C. D. Vecitis, K. R. Zodrow, S. Kang and M. Elimelech, ACS Nano, 2010, 4, 5471.

31 C.-Y. Chen and C. T. Jafvert, Environ. Sci. Technol., 2010, 44, 6674.

32 I. Fenoglio, M. Tomatis, D. Lison, J. Muller, A. Fonseca, J. B. Nagy and B. Fubini, Free Radical Biol. Med., 2006, 40, 1227.

33 K. Pulskamp, S. Diabaté and H. F. Krug, Toxicol. Lett., 2007, 168, 58.

34 Y. Tu, M. Lv, P. Xiu, T. Huynh, M. Zhang, M. Castelli, Z. Liu, Q. Huang, C. Fan, H. Fang and R. Zhou, Nat. Nanotechnol., 2013, 8, 594. 